\title{
Flap endonuclease 1 is a promising candidate biomarker in gastric cancer and is involved in cell proliferation and apoptosis
}

\author{
KEJIA WANG, CHUNHONG XIE and DAORONG CHEN \\ Department of Gastroenterology, The First Affiliated Hospital \\ of Chongqing Medical University, Chongqing 400016, P.R. China
}

Received November 30, 2013; Accepted February 20, 2014

DOI: $10.3892 /$ ijmm.2014.1682

\begin{abstract}
As a DNA repair protein, flap endonuclease 1 (FEN1), a structure-specific 5' nuclease, plays pivotal roles in the maturation of Okazaki fragments, long-patch base excision repair, restarting of stalled replication forks and telomere maintenance. FEN1 possesses 5' endonuclease, 5' exonuclease and gap-endonuclease activities, which render it an essential node in maintaining genome fidelity. The aim of this study was to investigate the association between the expression level of FEN1 and gastric cancer and to explore the role of FEN1 in carcinogenesis and the progression of gastric cancer. The mRNA and protein expression of FEN1 in 42 matched pairs of human gastric tumor tissues and corresponding normal tissues were measured by semiquantitative reverse transcription-PCR and immunohistochemical staining. FEN1 expression was downregulated in the SGC-7901 gastric cancer cells following transfection with siRNA targeting the FEN1 gene. Western blot analysis was used to evaluate the protein expression of FEN1 in SGC-7901 human gastric cancer cells in order to verify the transfection efficiency of FEN1 siRNA. Moreover, cell proliferation was analyzed by MTS assay. The apoptosis of the cells was determined by flow cytometry. Our results revealed that FEN1 was overexpressed in gastric cancer in comparison to the corresponding normal gastric tissues $(\mathrm{P}<0.01)$. We further confirmed that FEN1 expression has a positive correlation with the degree of differentiation $(\mathrm{P}=0.027)$, lymphatic metastasis $(\mathrm{P}=0.001)$, tumor size $(\mathrm{P}=0.026)$ and TNM stage $(\mathrm{P}=0.020)$ of gastric cancer. A high FEN1 expression in SGC-7901 cells can be effectively downregulated by siRNA constructed to target the FEN1 gene. Moreover, the inhibition of FEN1 expression suppressed the proliferation and induced the apoptosis of SGC-7901 cells. Taken together, our results indicate that FEN1
\end{abstract}

Correspondence to: Dr Daorong Chen, Department of Gastroenterology, The First Affiliated Hospital of Chongqing Medical University, 1st Youyi Road, Chongqing 400016, P.R. China

E-mail:63153820@qq.com; 993679065@qq.com

Key words: flap endonuclease 1, gastric cancer, siRNA, cell proliferation, cell apoptosis may be a promising biomarker for the diagnosis of gastric cancer and individual therapy.

\section{Introduction}

Gastric cancer has been proven to be one of the most common and most commonly diagnosed malignant tumors worldwide (1), with a low 5-year survival rate (2). As is already known, early diagnosis and effective therapeutic targets are critical factors influencing clinical treatment and prognosis. However, the underlying mechanisms of tumorigenesis and the progression of gastric cancer have not been fully elucidated.

Flapendonuclease-1 (FEN1), a structure-specific 5' nuclease (3), is a DNA replication protein which is currently accepted as a pivotal component of DNA molecule metabolism (4) due to its 5'-flap endonuclease (5), 5' exonuclease (6) and gapendonuclease activities $(7,8)$. It plays a vital role in Okazaki fragment maturation (9), the restarting of stalled replication forks $(7,10)$, telomere maintenance $(11,12)$, long-pace base excision repair (13) and apoptosis-induced fragmentation (7), which render it an essential node in maintaining genomic fidelity (14) and in preventing cells from carcinogenesis (4-14).

However, a growing body of evidence suggests that the abnormal expression of (FEN1) is involved in the initiation and progression of cancer and the development of disease (15-17). It has been reported that FEN1 is overexpressed in lung cancer cell lines (18), metastatic prostate cancer cell lines (19) and gastric cancer cell lines (20) compared with normal cell lines. In addition, the upregulated expression of FEN1 has been reported in tumor tissues, including prostate cancer (21), neuroblastoma (22), pancreatic cancer (23) and breast cancer (24) at the mRNA and protein level. FEN1 has further been confirmed to be upregulated in lung cancer, testicular cancer,glioblastoma and astrocytoma (25).

Therefore, in this study, the expression of FEN1 was evaluated in gastric tumor tissues by RT-PCR. In addition, the association between FEN1 expression and the clinicopathological characteristics of gastric cancer patients was explored by immunohistochemistry and regarded as evidence that FEN1 may be a potential biomarker for the diagnosis of gastric cancer. The effects of the silencing of FEN1 by siRNA on the proliferation and apoptosis of the selected gastric cell line were also evaluated in order to elucidate the underlying mechanisms responsible for the proression of gastric cancer, 
and to determine whether FEN1 is a potential therapeutic target in this disease.

\section{Materials and methods}

Preparation of human tissues and total RNA. Samples of surgically resected gastric tumors and corresponding normal tissues from 42 patients with gastric cancer were collected from the Department of Gastrointestinal Surgery, The First Affiliated Hospital of Chongqing Medical University, Chongqing, China, after obtaining written informed consent from each patient. These specimens were momentarily stored in liquid nitrogen for further research. All the gastric cancer patients were admitted to our hospital from February 2012 to April 2013. All 42 patients were determinately diagnosed by a pathological technique and had not received any pre-operative chemotherapy, radiotherapy or immunotherapy. The tumors obtained were divided into 6 groups according to the age ( $>65$ or $\leq 65$ years) and gender (male or female) of the patients, the differentiation grade (low, moderate or high), tumor size ( $>3 \mathrm{~cm}$ or $\leq 3 \mathrm{~cm}$ ), the lymphatic metastasis (positive or negative) and the TNM classification (I + II or III + IV) of tumors. The study was approved by the Ethics Committee of The First Affiliated Hospital of Chongqing Medical University. Total RNA was isolated from the tissues using RNAiso reagent (Takara Bio, Inc., Shiga, Japan). The concentration of the RNA was determined using a NanoDrop 2000 spectrophotometer (Thermo Fisher Scientific, Waltham, MA, USA).

Cell culture and transfection of cells with siRNA. Three gastric cancer cell lines, SGC-7901, MKN-28, MGC-803, were acquired from the Molecular Medicine and Cancer Research Center of Chongqing Medical University. All the cell lines were maintained in RPMI-1640 medium (Invitrogen, Grand Island, NY, USA) supplemented with $10 \%$ fetal bovine serum (Invitrogen), $1 \%$ penicillin-streptomycin and $2.0 \mathrm{~g} / 1$ sodium bicarbonate at $36.8^{\circ} \mathrm{C}$. The cells were used for the extraction of total RNA and protein when they reached $80-90 \%$ confluent. The siRNA vectors targeting FEN1 in the human gastric cells were designed and constructed by Invitrogen. A negative control (NC) siRNA was also used. The sequences of the siRNA were as follows: sense strand, 5'-GGACUUGUAGUCCUGCGAUTT-3' and antisense strand, 5'-AUCGCAGGACUACAAGUCCTT-3'. The cells were plated in 6 -well plates and each well had $3 \times 10^{5}$ cells. After $24 \mathrm{~h}$, the cells were cultured in an incubator containing $\mathrm{CO}_{2}$ at $36.7^{\circ} \mathrm{C}$, the mixture of siRNA and Lipofectamine ${ }^{\mathrm{TM}} 2000$ (Invitrogen) was added to each well containing cells and according to the manufacturer's instructions. Fresh medium was used to replace the transfection mixture after $6 \mathrm{~h}$. Forty-eight hours after transfection, the cells were used for further experiments.

Semiquantitative reverse transcription-PCR (RT-PCR). The mRNA levels of FEN1 in the tumor tissues and corresponding normal tissues were examined by RT-PCR. Total mRNA from all the tissues was reverse transcribed into complementary DNA using a reverse transcription kit (Takara Bio, Inc.) according to the manufacturer's instructions. The specific oligonucleotide primers used for FEN1 and those of the internal reference gene, $\beta$-actin, were designed using
Primer 5.0 software (Premier Biosoft, Palo Alto, CA, USA) and synthesized by Takara Bio, Inc.. The primers for FEN1 were as follows: forward, 5'-AGCCCGTGTATGTCTTTG-3' and reverse, 5'-AGTCAGGTGTCGCATTAG-3' and for $\beta$-actin forward, 5'-CCTTCTACAATGAGCTGCGT-3' and reverse, 5'-CCTGGATAGCAACGTACATG-3'. The PCR products were electrophoresed on $1.5 \%$ agarose gels. The expression of FEN1 relative to $\beta$-actin was measured using a Bio-Rad gel imaging analysis system and analyzed using Quantity One software (Bio-Rad, Hercules, CA, USA). The RT-PCR experiments were repeated independently 3 times.

Immunohistochemistry. Immunohistochemical staining was performed on paraffin-embedded tissues from 42 gastric cancer patients. The tissue sections were dewaxed with xylene and rehydrated with alcohol after baking in the $60^{\circ} \mathrm{C}$ thermostat for $1 \mathrm{~h}$. After rinsing in PBS, the sections were heated with citrate buffer in a microwave oven for antigen retrieval. Normal goat serum was then used to block out non-specific binding after washing in PBS, the endogenous peroxidase activity of the tissues had been already blocked by $3 \%$ peroxide. The cells were incubated with primary anti-FEN1 antibody (Epitomics, Burlingame, CA, USA) overnight at a dilution of 1:300 at $4^{\circ} \mathrm{C}$. The sections, after a thorough cleaning in PBS, were subjected to 1-h incubation with anti-rabbit secondary antibodies and were then incubated with horseradish peroxidase (HRP)-labeled streptavidin for $30 \mathrm{~min}$. In addition, the tissue sections were stained with diaminobenzidine (DAB) and then redyed by hematoxylin counterstain. Ultimately, the treated sections were observed under a microscope and estimated by the standard described in a previous study (24). The staining was scored according to two standards of FEN1 expression: the staining intensity scored from grade 0 to 3 ( 0 , negative; 1 , pale yellow staining; 2 , moderate yellow staining; 3 , brown staining) and the percentage of positively stained cells $(0$, no FEN1-positive cells, $1,<10 \%$ positive cells; $2,11-15 \%$ positive cells; $3,51-80 \%$ positive cells and $4,>80 \%$ positive cells). The final result of FEN1 expression was the product of scores of the staining intensity and the percentage of positively stained cells (negative, $0-1$; weakly positive, 1-2; moderately positive, 2-3; strongly positive, $\geq 3$ ).

Western blot analysis. Western blot analysis was used to verify the inhibitory effects of FEN1 siRNA. The 3 gastric cell lines were lysed using cell lysis buffer for the isolation of total protein. The bicinchoninic acid (BCA) protein assay kit was used to measured the concentration of the extracted protein of which $30 \mu \mathrm{l}$ was separated on SDS-PAGE for electrophoresis and electrophoretically transferred onto polyvinylidene fluoride (PVDF) membranes. The membranes were blocked in 5\% fat-free milk at room temperature and incubated for $2 \mathrm{~h}$ with a 1:1,000 dilution of primary rabbit monoclonal antibodies, followed by HRP-conjugated secondary antibody. After rinsing, the blots were detected using a chemiluminescence western blotting detection system. The western blot analysis experiments were repeated independently 3 times.

Detection of cell proliferation by MTS assay. The cells in the experimental group and control group were trypsinized and placed into 96-well culture plants in the logarithmic phase 


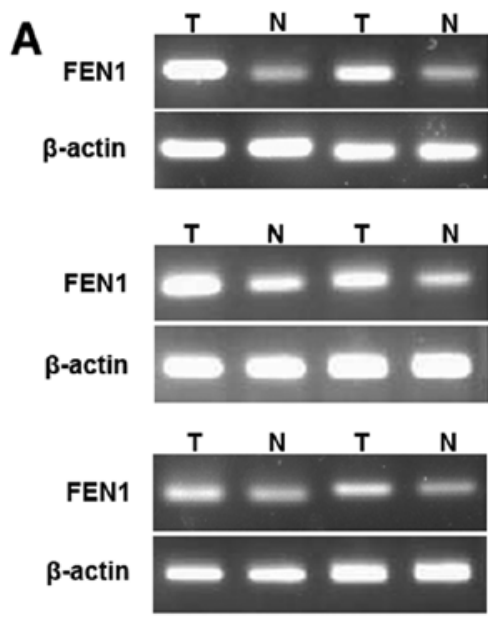

B

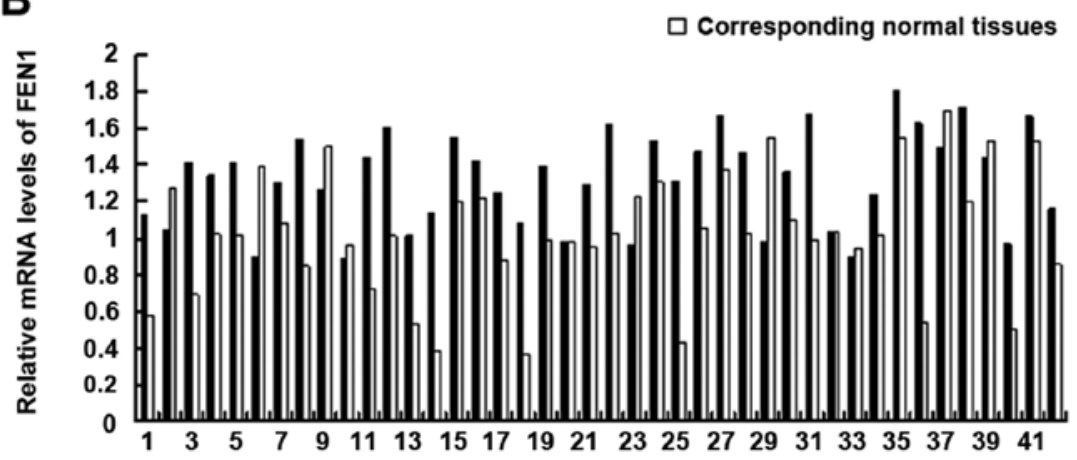

Figure 1. Expression of the flap endonuclease 1 (FEN1) gene in gastric tumor and corresponding normal tissues. Total RNA extracted from the 42 gastric tumor tissues and matched normal tissues was examined by semiquantitative reverse transcription-PCR (RT-PCR); $\beta$-actin was used as an internal reference. (A) Semiquantitative RT-PCR results of 6 out of 42 paired gastric tumor tissues. (B) Expression levels of FEN1 in 42 matched normal and tumor gastric cancer tissues. The relative mRNA expression levels of FEN1 were analyzed by Quantity One software relative to the value of $\beta$-actin. A higher expression of FEN1 was detected in 32 tumor tissues in comparison to the corresponding normal tissues among the 42 cases. (C) The differences between the gastric tumor tissues and the matched normal tissues were statistically significant. The data were evaluated by paired t-tests. ${ }^{* *} \mathrm{P}<0.01$.

at a density of $7 \times 10^{3}$ cells/well. The MTS kit (CellTiter 96 AQueous one solution reagent; Promega Corp., Madison, WI, USA) was then added to each well and the cells were incubated for a further $4 \mathrm{~h}$ before harvesting at $24,48,72$ and $96 \mathrm{~h}$ according to the manufacture's instructions. Optical density was measured at $490 \mathrm{~nm}$ for the absorbance values. These procedures were repeated independently 3 times.

Cell apoptosis assay. The fluorescein-isothiocyanate-labeled enhanced AnnexinV/propidium iodide Apoptosis Detection kit (Invitrogen) was used to determine the apoptotic rate of the cells in the experimental group and control group according to the manufacture's instructions. Methotrexate (MTX; Shanghai Yuanye Bio-Technology Co., Ltd, Shanghai, China) was used to induce cell apoptosis. The cells were then subjected to flow cytometry within $1 \mathrm{~h}$.

Statistical analysis. All the data were analyzed with a paired t-test, Wilcoxon rank sum test and $\chi^{2}$ test using IBM SPSS 19.0 software (SPSS Inc., Chicago, IL, USA). A value of $\mathrm{P}<0.05$ was considered to indicate a statistically significant difference.

\section{Results}

Expression of FEN1 gene in tumors compared with matched normal tissues. The FEN1 mRNA expression levels in the gastric tumor and corresponding normal tissues was determined by semiquantitative RT-PCR and the results are shown in Fig. 1. The FEN1 mRNA expression of 6 out of 42 paired gastric tumor tissues is shown in Fig. 1A. Of the 42 samples, $32(76 \%)$ displayed a higher FEN1 expression in the tumor tissues compared to the corresponding normal tissues (Fig. 1B). The relative mRNA expression of FEN1 in the tumor tissues and matched normal tissues was $1.32 \pm 0.26$ and $1.02 \pm 0.34$, respectively. The difference between the 2 groups (corresponding to normal and tumor tissues) was statistically significant $(\mathrm{P}<0.01)$ as shown in Fig. $1 \mathrm{C}$.

The relative protein expression of FEN1 in the 42 paired tissues was determined by immunohistochemistry. As shown in Fig. 2, FEN1 was primarily expressed in the nucleus and occasionally, in the cytoplasm. As shown in Table I, 32 samples of the 42 tumor tissues (76\%) and 8 of the 42 matched normal tissues (19\%) had a positive expression of FEN1. A higher 


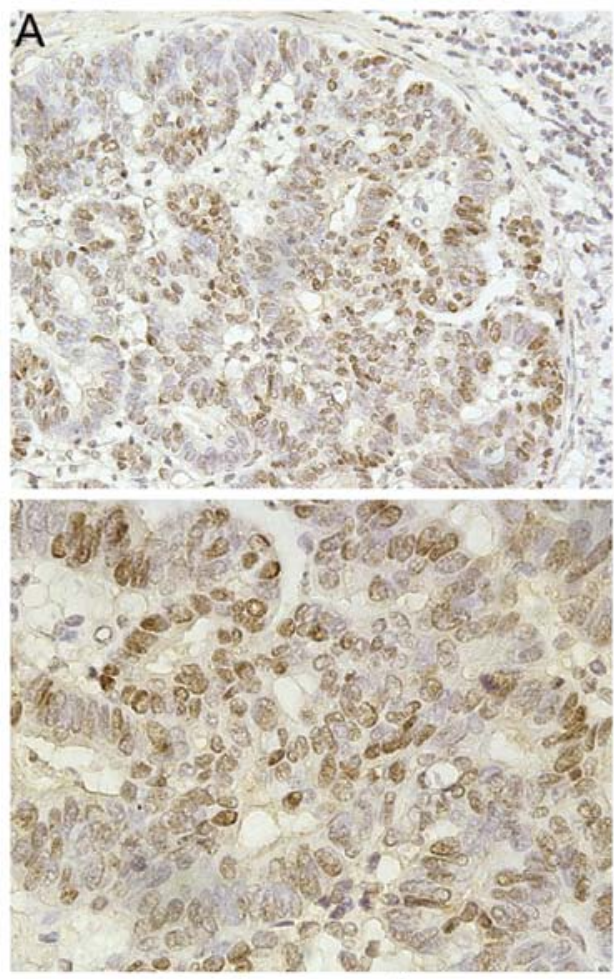

Gastric tumor tissue

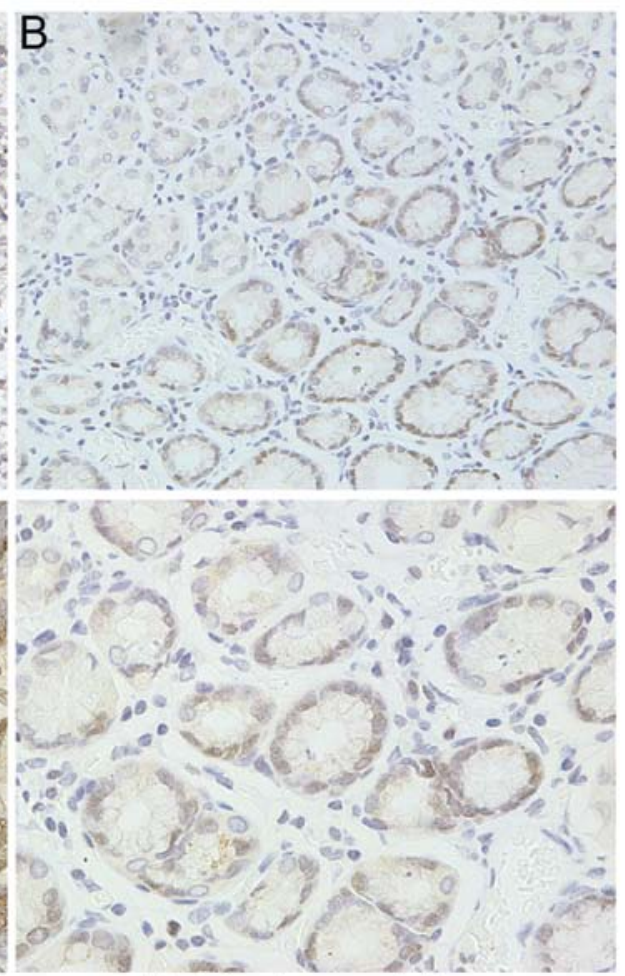

Matched normal tissue

Figure 2. Immunohistochemical analysis of a matched sample of gastric tumor and normal tissue using anti-flap endonuclease 1 (anti-FEN1) antibody. (A) Positive FEN1 expression in gastric tumor tissue (top panel, x200 magnification; bottom panel, x400 magnification). (B) Weakly positive FEN1 expression in normal tissues (top panel, x200 magnification; bottom panel, x400 magnification).

Table I. Expression of FEN1 in gastric cancer and matched normal tissues.

\begin{tabular}{lcccc}
\hline & & \multicolumn{2}{c}{ FEN1 expression } & \\
\cline { 3 - 4 } Group & Cases & Negative & Positive & P-value \\
\hline Tumor tissues & 42 & 10 & 32 & \\
Matched normal tissues & 42 & 34 & 8 & \\
\hline
\end{tabular}

FEN1 expression was determined by immunohistochemistry and the difference between the 2 groups was estimated by a Wilcoxon rank sum test. ${ }^{a} \mathrm{P}<0.01$ indicates statistical significance.

expression of FEN1 was observed in the tumor tissues in comparison to the corresponding normal tissues and the difference was statistically significant as determined by the Wilcoxon rank sum test $(\mathrm{P}<0.01)$.

Association between FEN1 expression and clinicopathological characteristics. The results of immunohistochemical staining and the clinicopathological characteristics of all the patients are presented in Table II. We observed a significant difference between the groups (positive or negative for FEN1 expression) as regards the degree of differentiation $(\mathrm{P}=0.027)$, lymphatic metastasis $(\mathrm{P}=0.001)$, tumor size $(\mathrm{P}=0.026)$ and $\mathrm{TNM}$ stage $(\mathrm{P}=0.020)$. However, as regards age and gender, no significant differences were observed between the groups $(\mathrm{P}>0.05)$. The outcomes intimated that the expression of FEN1 positively correlates with the degree of differentiation, lymphatic metastasis, tumor size and TNM stage in gastric cancer, but has no significant correlation with age and gender, as shown in our 42 cases of gastric cancer.

FEN1 expression is markedly inhibited by siRNA in gastric cancer cells (SGC-7901). In order to validate the inhibitory effects of FEN1 siRNA, the expression levels of FEN1 in the SGC-7901 cells were determined by western blot analysis. As shown in Fig. 3, the downregulation of FEN1 expression was observed in the SGC-7901 cells transfected with FEN1 siRNA compared to the cells in the negative control group (transfected with NC siRNA; $\mathrm{P}<0.01$ ). These results demonstrated that FEN1 expression in the SGC-7901 cells was markedly decreased following transfection with FEN1 siRNA.

Downregulation of FEN1 expression suppresses SGC-7901 cell proliferation. The proliferation of the SGC-7901 cells transfected with FEN1 siRNA was determined to be markedly suppressed at 24, 48, 72 and $96 \mathrm{~h}$ in comparison to the cells transfected with the negative control (NC siRNA). The data from MTS assay revealed that the proliferation of the SGC-7901 gastric cancer cells was suppressed following the downregulation of FEN1 gene expression by siRNA (Fig. 4).

Downregulation of FEN1 expression induces the apoptosis of $S G C-7901$ cells. The apoptotic rates of the 2 groups of cells 
Table II. Association between FEN1 expression and the clinicopathological characteristics in gastric cancer patients.

\begin{tabular}{|c|c|c|c|c|}
\hline \multirow[b]{2}{*}{ Variable } & \multirow[b]{2}{*}{ Cases } & \multicolumn{2}{|c|}{ FEN1 } & \multirow[b]{2}{*}{ P-value } \\
\hline & & Positive cases (\%) & Negative cases (\%) & \\
\hline Total cases & 42 & $32(76.2)$ & $10(23.8)$ & \\
\hline Age (years) & & & & $>0.05$ \\
\hline$>65$ & 15 & $12(80)$ & $3(20)$ & \\
\hline$\leq 65$ & 27 & $20(74.1)$ & $7(25.9)$ & \\
\hline Gender & & & & $>0.05$ \\
\hline Male & 26 & $20(76.9)$ & $6(23.1)$ & \\
\hline Female & 16 & $12(75)$ & $4(25)$ & \\
\hline Degree of differentiation & & & & 0.027 \\
\hline High-moderate & 16 & $9(56.1)$ & $7(43.8)$ & \\
\hline Low & 26 & $23(88.5)$ & $3(11.5)$ & \\
\hline Lymphatic metastasis & & & & 0.001 \\
\hline Negative & 18 & $9(50)$ & $9(50)$ & \\
\hline Positive & 24 & $23(95.8)$ & $1(4.2)$ & \\
\hline Tumor size $(\mathrm{cm})$ & & & & 0.026 \\
\hline$>3$ & 23 & $21(91.3)$ & $2(8.7)$ & \\
\hline$\leq 3$ & 19 & $11(57.9)$ & $8(42.1)$ & \\
\hline TNM stage & & & & 0.020 \\
\hline $\mathrm{I}+\mathrm{II}$ & 15 & $8(53.3)$ & $7(46.7)$ & \\
\hline III + IV & 27 & $24(88.9)$ & $3(11.1)$ & \\
\hline
\end{tabular}

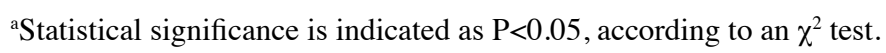

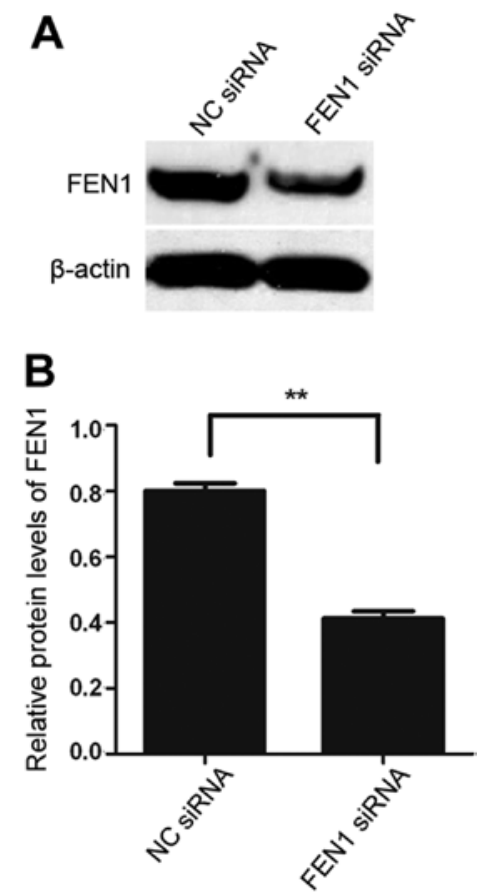

Figure 3. Results of the silencing of flap endonuclease 1 (FEN1) by siRNA in the SGC-7901 gastric cancer cell line. (A) FEN1 expression was significantly inhibited by FEN1 siRNA in SGC-7901 cells. The SGC-7901 cells were transfected with FEN1 siRNA or negative control (NC) siRNA. FEN1 expression was measured by western blot analysis $48 \mathrm{~h}$ after transfection. $\beta$-actin was used as an internal control. (B) The data were determined using Quantity One software and estimated by a paired t-test. ${ }^{* *} \mathrm{P}<0.01$.

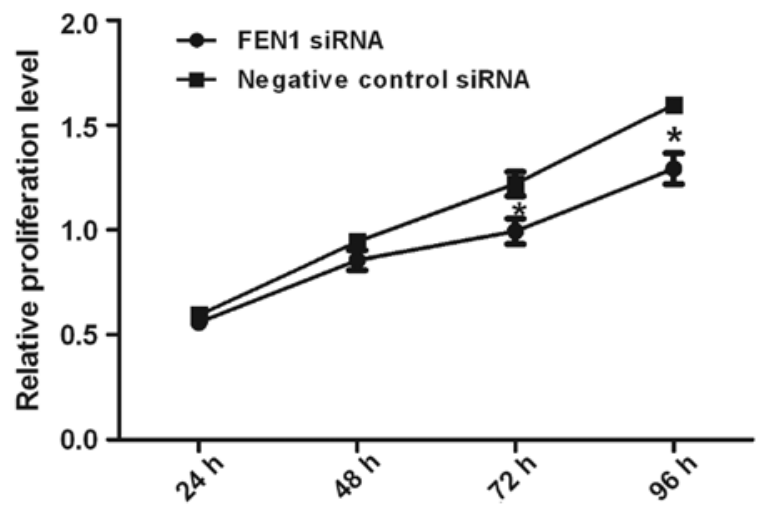

Figure 4. Downregulation of flap endonuclease 1 (FEN1) suppresses the proliferation of SGC-7901 cells. Effects of the downregulation of FEN1 expression on cell proliferation in SGC-7901 cells transfected with FEN1 siRNA or negative control (NC) siRNA were determined by MTS assay at $24,48,72$ and $96 \mathrm{~h}$. Data shown are the means of 3 experiments. ${ }^{*} \mathrm{P}<0.05$.

were detected by flow cytometry, and the results demonstrated that the average apoptotis rate of the SGC-7901 cells transfected with FEN1 siRNA was markedly increased in comparison to the cells in the negative control group (transfected with NC siRNA; $\mathrm{P}<0.01)$. The outcomes intimated that the downregulation of FEN1 expression induced the apoptosis of SGC-7901 gastric cancer cells (Fig. 5). 

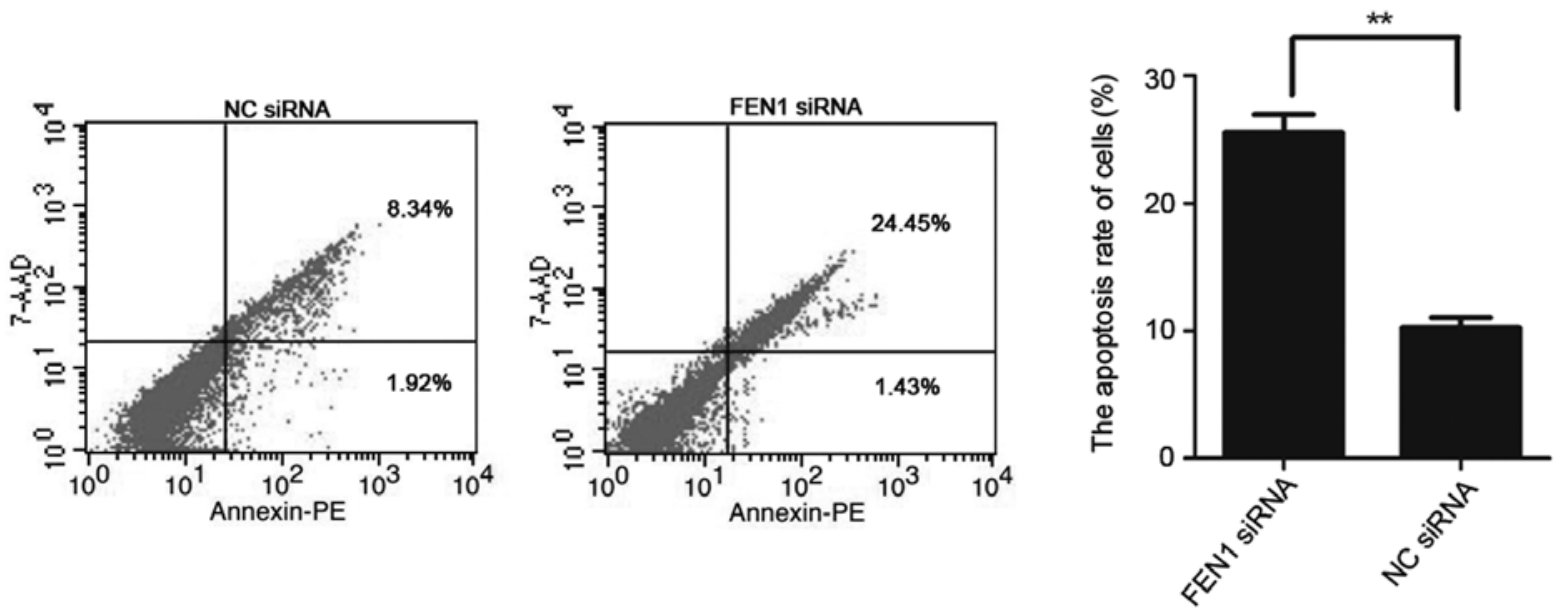

Figure 5. Downregulation of flap endonuclease 1 (FEN1) induces the apoptosis of SGC-7901 cells. The SGC-7901 cells were harvested and subjected to flow cytometry following treatment with MTX for $48 \mathrm{~h}$. The apoptotic rate of the cells transfected with FEN1 siRNA or negative control (NC) siRNA was measured by flow cytometry. The average apoptotic rate of the SGC-7901 cells transfected with FEN1 siRNA was significantly increased. ${ }^{* *} \mathrm{P}<0.01$.

\section{Discussion}

As a structure-specific endonuclease (3), FEN1 has been confirmed to be a DNA replication protein related to the coordination of various fundamental DNA transactions (26) and the prevention of cell carcinogenesis (4-14). Therefore, FEN1 is widely considered as an effective tumor suppressor (9) involved in the replication and apoptosis of DNA. However, accumulating evidence indicates the existance of a significant association between the abnormal expression of FEN1 and the development of cancer or the progression of pathological transformation. Although the overexpression of FEN1 is being increasingly reported in diverse types of cancer, including stomach tumors mentioned in a few studies (24), its correlation with the clinicopathological characteristics of gastric cancer patienits remains unelucidated. Thus, in this study, we first determined the expression of FEN1 in 42 paired tumor and normal tissues at the mRNA and protein levels to validate FEN1 expression in gastric tumors. The results demonstrated that the expression level of FEN1 was evidently greater in gastric tumor tissues compared with the corresponding normal tissues. This is consistent with previously reported data from a gene expression profiling array revealing the differences in FEN1 expression between cancer tissues and matched normal tissues (24). Furthermore, the data obtained in the present study demonstrate that the overexpression of FEN1 is associated with tumor size, lymphatic metastasis, degree of differentiation and TNM stage in gastric cancer, whereas it is not associated with age and gender (Table II). These data suggest that the DNA repair gene, FEN1, may be a more precise maker for identifying gastric cancer with clinical data and selecting patients in different situations. Owing to its involvement in DNA replication, restoration and degradation, the increased expression of FEN1 is also a probable response to growing DNA damage in cancer cells.

The increased expression of FEN1 has not only been detected in various types of tumor tissues, but also in cancer cell lines $(18,19)$ including gastric cancer cells $(20)$. It has been reported that FEN1 may also be conductive to telomere mainte- nance in human cells $(11,12,27)$. Thus the dysfunction of FEN1 in telomere and genomic stability may participate in the transformation of normal cells into cancer cells. In addition, FEN1 has been detected in all proliferative cells, but in dormant cells it has only been detected at a very low level (28). A previous study highlighted that FEN1 participates in the progression of mouse gastrointestinal tract cancer in the form of haploinsufficiency (15). This suggests that FEN1 may be necessary in the growth and progression of tumors. The expression level of FEN1 increases with tumor grade and dedifferentiation $(21,24)$. In contrast to its role as an effective tumor suppressor, it is presumed that the increased expression of FEN1 contributes to the hyper-proliferation of tumor cells (29).

Therefore, in this study, to further explore the effects of FEN1 on proliferation and apoptosis in carcinoma cell lines in vitro, we downregulated FEN1 using siRNA in the SGC-7901 gastric cancer cell line and determined this downregution by western blot analysis. Our results revealed that the cell growth of FEN1-silenced cells was markedly inhibited and the apoptotic rate of these cells was significantly greater in comparison with the cells in the negative control group (transfected with NC siRNA), indicating that the downregulatiion of FEN1 in SGC-7901 gastric cancer cells suppressed proliferation and induced apoptosis. These data suggest that FEN1 may be an effective therapeutic target in human gastric cancer. Several FEN1 inhibitors, including 2,4-diketobutyric acids and N-hydroxyurea series, were discovered and have been developed over the years, permitting sensitization to DNA injury agents $(30,31)$. These may be promising treatment methods that could enhance the traditional chemotherapeutics for stomach and other types of cancer.

In conclusion, our results revealed that FEN1 was overexpressed in gastric cancer in comparison with corresponding normal tissues, and the high expression of FEN1 positively correlated with tumor size, lymphatic metastasis, degree of differentiation and TNM stage in gastric cancer. Moreover, the downregulation of FEN1 suppressed the proliferation and induced the apoptosis of SGC-7901 gastric carcinoma cells. Therefore, FEN1 may be used as an effective biomarker for 
the diagnosis and treatment of gastric cancer. To the best of our knowledge, ours is the first study to report the association between FEN1 expression and the clinicopathological characteristics of gastric cancer patients, as well as the effects of silencing FEN1 on the proliferation and apoptosis of SGC-7901 gastric cancer cells.

\section{Acknowledgements}

We appreciate the assistance provided by the Department of Gastrointestinal Surgery, The First Affiliated Hospital of Chongqing Medical University. This study was supported by the Research Fund of Chongqing Municipal Health Bureau (grant no. 2009-2-345).

\section{References}

1. Mitelman F: Catalogue of Chromosomes Aberrations in Cancer Cytogenet Cell Genet 36: 1-515, 1983.

2. Hartgrink HH, Jansen EPM, van Grieken NCT and van de Velde CJH: Gastric cancer-Authors' reply. Lancet 374: 1594-1595, 2009.

3. Harrington JJ and Lieber MR: The characterization of a mammalian DNA structure-specific endonuclease. EMBO J 13 : 1235-1246, 1994.

4. Liu Y, Kao HI and Bambara RA: Flap endonuclease 1: a central component of DNA metabolism. Ann Rev Biochem 73: 589-615, 2004

5. Frank G, Qiu J, Somsouk M, et al: Partial functional deficiency of E160D flap endonuclease-1 mutant in vitro and in vivo is due to defective cleavage of DNA substrates. J Biol Chem 273 33064-33072, 1998.

6. Shen B, Singh P, Liu R, et al: Multiple but dissectible functions of FEN-1 nucleases in nucleic acid processing, genome stability and diseases. Bioessays 27: 717-729, 2005.

7. Zheng L, Zhou M, Chai Q, et al: Novel function of the flap endonuclease 1 complex in processing stalled DNA replication forks. EMBO Rep 6: 83-89, 2005.

8. Tsutakawa SE, Classen S, Chapados BR, et al: Human flap endonuclease structures, DNA double-base flipping, and a unified understanding of the FEN1 superfamily. Cell 145: 198-211, 2011.

9. Henneke G, Friedrich-Heineken E and Hübscher U: Flap endonuclease 1: a novel tumour suppressor protein. Trends Biochem Sci 28: 384-390, 2003.

10. Saharia A, Teasley DC, Duxin JP, Dao B, Chiappinelli KB and Stewart SA: FEN1 ensures telomere stability by facilitating replication fork re-initiation. J Biol Chem 285: 27057-27066, 2010.

11. Saharia A, Guittat L, Crocker S, et al: Flap endonuclease 1 contributes to telomere stability. Curr Biol 18: 496-500, 2008.

12. Sampathi S, Bhusari A, Shen B and Chai W: Human flap endonuclease $\mathrm{I}$ is in complex with telomerase and is required for telomerase-mediated telomere maintenance. J Biol Chem 284: 3682-3690, 2009.

13. Klungland A and Lindahl T: Second pathway for completion of human DNA base excision-repair: reconstitution with purified proteins and requirement for DNase IV (FEN1). EMBO J 16: 3341-3348, 1997.
14. Singh P, Zheng L, Chavez V, Qiu J and Shen B: Concerted action of exonuclease and Gap-dependent endonuclease activities of FEN-1 contributes to the resolution of triplet repeat sequences (CTG)n- and (GAA)n-derived secondary structures formed during maturation of Okazaki fragments. J Biol Chem 282: 3465-3477, 2007.

15. Kucherlapati M, Yang K, Kuraguchi M, et al: Haploinsufficiency of Flap endonuclease (Fen1) leads to rapid tumor progression. Proc Natl Acad Sci USA 99: 9924-9929, 2002.

16. Zheng L, Dai H, Zhou M, et al: Fen1 mutations result in autoimmunity, chronic inflammation and cancers. Nat Med 13: 812-819, 2007.

17. Larsen E, Kleppa L, Meza TJ, et al: Early-onset lymphoma and extensive embryonic apoptosis in two domain-specific Fen1 mice mutants. Cancer Res 68: 4571-4579, 2008.

18. Sato M, Girard L, Sekine I, et al: Increased expression and no mutation of the Flap endonuclease (FEN1) gene in human lung cancer. Oncogene 22: 7243-7246, 2003.

19. LaTulippe E, Satagopan J, Smith A, et al: Comprehensive gene expression analysis of prostate cancer reveals distinct transcriptional programs associated with metastatic disease. Cancer Res 62: 4499-4506, 2002.

20. Kim JM, Sohn HY, Yoon SY, et al: Identification of gastric cancer-related genes using a cDNA microarray containing novel expressed sequence tags expressed in gastric cancer cells. Clin Cancer Res 11: 473-482, 2005.

21. Lam JS, Seligson DB, Yu H, et al: Flap endonuclease 1 is overexpressed in prostate cancer and is associated with a high Gleason score. BJU Int 98: 445-451, 2006.

22. Krause A, Combaret V, Iacono I, et al: Genome-wide analysis of gene expression in neuroblastomas detected by mass screening. Cancer Lett 225: 111-120, 2005.

23. Iacobuzio-Donahue CA, Maitra A, Olsen M, et al: Exploration of global gene expression patterns in pancreatic adenocarcinoma using cDNA microarrays. Am J Pathol 162: 1151-1162, 2003.

24. Singh P, Yang M, Dai H, et al: Overexpression and hypomethylation of flap endonuclease 1 gene in breast and other cancers. Mol Cancer Res 6: 1710-1717, 2008

25. Nikolova T, Christmann M and Kaina B: FEN1 is overexpressed in testis, lung and brain tumors. Anticancer Res 29: 2453-2459, 2009.

26. Balakrishnan L and Bambara RA: Flap Endonuclease 1. Ann Rev Biochem 82: 119-138, 2013.

27. Saharia A and Stewart S: FEN1 contributes to telomere stability in ALT-positive tumor cells. Oncogene 28: 1162-1167, 2009.

28. Kim IS, Lee MY, Lee IH, Shin SL and Lee SY: Gene expression of flap endonuclease-1 during cell proliferation and differentiation. Biochim Biophys Acta 1496: 333-340, 2000.

29. Zheng L, Jia J, Finger LD, Guo Z, Zer C and Shen B: Functional regulation of FEN1 nuclease and its link to cancer. Nucleic Acids Res 39: 781-794, 2011.

30. Tumey LN, Huck B, Gleason E, et al: The identification and optimization of 2,4-diketobutyric acids as flap endonuclease 1 inhibitors. Bioorg Med Chem Lett 14: 4915-4918, 2004.

31. Tumey LN, Bom D, Huck B, et al: The identification and optimization of a $\mathrm{N}$-hydroxy urea series of flap endonuclease 1 inhibitors. Bioorg Med Chem Lett 15: 277-281, 2005. 